\title{
Performance of Fly Visual Interneurons during Object Fixation
}

\author{
Bernd Kimmerle and Martin Egelhaaf \\ Lehrstuhl für Neurobiologie, Fakultät für Biologie, Universität Bielefeld, D-33501 Bielefeld, Germany
}

\begin{abstract}
Neurons involved in the processing of optic flow are usually analyzed using stimuli designed by the experimenter. However, in real life optic flow depends on locomotive behavior. We characterized the performance of motion-sensitive neurons in the visual system of the fly using optic flow as occurring in behavioral situations during object fixation. Optic flow generated by tethered flying flies in a flight simulator was subsequently replayed while recording the responses of two cell types in the fly's motion pathway presumably involved in the detection of objects and of deviations from a straight flight course, respectively. FD1b cells, which are representatives of the so-called figure-detection cells, responded very specifically to object motion. Although object selectivity of these cells is attributable to inhibition during largefield motion, the influence of background motion during object
\end{abstract}

fixation was almost negligible. In contrast, the cells of the socalled horizontal system (HS cells) are most sensitive to background motion, as elicited during deviations of the animal from its course. During object fixation, the responses of HS cells depended on both object and background motion. The simulated distance of the background to the fly did not have a strong influence on the responses of either cell type. The specificity for detecting deviations from a straight course is enhanced by subtraction of the signals of HS cells in both halves of the brain. In contrast, the FD1b cells in the two halves of the brain need to interact in a nonlinear way to ensure efficient detection of objects.

Key words: behaviorally relevant stimuli; figure-ground discrimination; insect; object fixation; optic flow; visual system
During locomotion the retinal image is subjected to continuous changes. This "optic flow" is shaped by the direction and speed of locomotion. During translational motion optic flow also depends on the distance of the objects in the surround. When passing closer objects, they appear to move faster than more distant objects. Visual systems use relative motion cues for object detection. Neurons tuned to relative motion between an object and its background have been found e.g., in pigeons (Frost and Nakayama, 1983), cats (Sterling and Wickelgren, 1969), monkeys (Allman et al., 1985; Tanaka et al., 1986), hawkmoths (Collett, 1971), and flies (Egelhaaf, 1985b; Gauck and Borst, 1999). All these neurons are excited by object motion and inhibited by background motion.

How specifically relative motion-sensitive neurons respond to objects in behavioral situations in which optic flow depends on the animal's locomotive behavior will be analyzed in the fly visual system. The fly displays virtuosic visually guided orientation behavior (Land and Collett, 1974; Wagner, 1982, 1986; Borst, 1990; Kimmerle et al., 1996) that can be analyzed under controlled stimulus conditions in a flight simulator (Virsik and Reichardt, 1976; Reichardt and Poggio, 1979; Egelhaaf, 1985a; Kimmerle et al., 1997). Moreover, large motion-sensitive neurons [tangential cells (TCs)] are known that are sensitive to various aspects of optic flow. Two classes of TCs, the FD cells and the HS cells, are involved in evaluating optic flow during locomotion in the horizontal plane. Both cell classes spatially integrate local motion information over large parts of the visual field. In addition, FD cells are inhibited by background motion and thus respond strongest to object motion (Egelhaaf, 1985b; Gauck and Borst, 1999; Kimmerle and Egelhaaf, 2000). HS cells lack this kind of inhibition and are maximally excited during global horizontal motion as induced during turns of the fly around its vertical body axis, i.e., when the fly deviates from a straight course (Hausen, 1982a,b). Both cell types

Received Feb. 15, 2000; revised May 5, 2000; accepted May 31, 2000.

This work was supported by the Deutsche Forschungsgemeinschaft. We are grateful to C.G. Galizia, R. Kern, R. Kurtz, and A.-K. Warzecha and to two anonymous referees for helpful comments on this manuscript.

Correspondence should be addressed to Martin Egelhaaf, Lehrstuhl für Neurobiologie, Fakultät für Biologie, Universität Bielefeld, Postfach 1001 31, D-33501 Bielefeld, Germany. E-mail: martin.egelhaaf@biologie.uni-bielefeld.de.

Dr. Kimmerle's present address: Institut für Neurobiologie, Freie Universität Berlin, Königin-Luise-Strasse 28-30, D-14195 Berlin, Germany.

Copyright (C) 2000 Society for Neuroscience $0270-6474 / 00 / 206256-11 \$ 15.00 / 0$ are likely to play an essential role in visually guided orientation behavior, FD cells in mediating object detection and fixation and HS cells in stabilizing the flight course against disturbances (for review, see Hausen and Egelhaaf, 1989; Egelhaaf and Borst, 1993; Egelhaaf and Warzecha, 1999).

We investigated the responses of one type of FD cells, the FD1b cells and of HS cells to optic flow as experienced by a fly during object fixation. The motion stimuli were generated in previous behavioral experiments with tethered flying flies in a flight simulator (Kimmerle et al., 2000). In these experiments, flies were confronted with optic flow simulating translational flight in a surround consisting of an object and its background. The turning responses of the fly influenced the visual input in a similar way as in free flight. In the present study optic flow generated in this way was replayed while recording the activity of FD1b cells (Kimmerle and Egelhaaf, 2000) and of HS cells. Modifying specific aspects of the original optic flow helped us to determine the influence of object and background motion on the cellular responses.

\section{MATERIALS AND METHODS}

Animal preparation. Blowflies of the genus Lucilia were obtained from laboratory stocks. All experiments were done on female flies. For intracellular recordings flies were used that had hatched not more than $2 \mathrm{~d}$ before the experiment. The flies taken for extracellular recordings were usually older than $2 \mathrm{~d}$. Animals were prepared as reported previously (Kimmerle and Egelhaaf, 2000). In short, the rear surface of the head capsule was opened to get access to the right optic lobe from posterior. The head capsule was supplied with Ringer's solution. To avoid movements, the proboscis was cut, and the gut was pulled out from behind. The antennae were removed, and the antennal muscles were cut. Some of the neck muscles were severed. In most preparations the abdomen was opened, and the heart was removed. The abdomen was then filled with Ringer's solution. The wounds were sealed with wax. The animals were adjusted in the setup by aligning the eye equator in a horizontal plane. Electrophysiological recordings were always performed in the right optic lobe.

Recording techniques. All FD1b cells were recorded extracellularly with glass electrodes (Hilgenberg or Clark; outer/inner diameter: 1.5/1.17 mm). Pulled on a vertical puller (Getra, Munich, Germany) and filled with $1 \mathrm{M}$ $\mathrm{KCl}$ solution, the electrodes had resistances of 4-8 $\mathrm{M} \Omega$. A wide tip electrode filled with Ringer's solution and connected to a syringe was used as an indifferent electrode and to control solution supply to the head capsule. The recorded signal was bandpass-filtered and amplified with standard electrophysiological equipment (built by the electronic workshop of the Max-Planck-Institut (MPI) für biologische Kybernetik, Tübingen, Germany). Spikes were transformed into pulses of fixed height and duration and fed into a personal computer (PC) via the digital or the analogto-digital (A/D) port of an I/O card (DT2801 A; Data Translation, 
Marlboro, MA) at a sampling rate of $1 \mathrm{kHz}$. Most of the recordings were additionally stored on DAT (recorder: DTC-670; Sony, Tokyo, Japan). In these cases spike discrimination was performed off-line. All HS cells were recorded intracellularly with glass electrodes (GC100TF-10; Clark, Edenbridge, UK). Pulled on a Brown/Flaming puller (P97; Sutter Instruments, San Rafael, CA) and filled with $1 \mathrm{~m} \mathrm{KCl}$ solution, the electrodes had resistances of 40-90 $\mathrm{M} \Omega$. Indifferent electrodes were the same as in the extracellular recordings. To confirm identification of the HS cells by functional criteria (see below), in some experiments the cells were stained iontophoretically (current, approximately $-1 \mathrm{nA}$ ). In these cases the electrodes were filled with a solution of Lucifer yellow (Sigma, Deisenhofen, Germany) in $1 \mathrm{~m} \mathrm{LiCl}$. The stained cells were examined in the living animal without further dissection under a fluorescence microscope (Orthoplan; Leitz, Wetzlar, Germany). The recorded signal was amplified 10 -fold with standard electrophysiological equipment (built by the electronic workshop of the MPI für biologische Kybernetik, Tübingen, Germany), fed into a PC via the A/D-port of an I/O-card (DT2801 A; Data Translation), and stored at a sampling rate of $1 \mathrm{kHz}$. The programs for stimulus control and data acquisition were written in C (Borland, Scotts Valley, CA).

Identification of cells. FD cells are sensitive to horizontal motion and respond stronger to small objects moving in their preferred direction than to large-field motion in extended parts of the visual field ("small-fieldtuning"). They were originally subdivided into four response types according to their preferred direction of motion, the location of their excitatory receptive field, and the direction selectivity of their contralateral inhibitory input (Egelhaaf, 1985b). Cells recorded in the present study were classified as FD1b (Kimmerle and Egelhaaf, 2000) if they satisfied the following criteria: (1) They exhibited small-field-tuning. (2) Their preferred direction of motion was front-to-back. (3) Their receptive field was centered in the frontal part of the visual field. (4) They were inhibited by both contralateral back-to-front motion and, less strongly, by contralateral frontto-back motion. The recording site for FD1b cells was located in the central region of the lobula plate. Recording electrodes were positioned using tracheae as landmarks. Recording times lasted up to $\sim 90$ min. By functional criteria alone it cannot be ensured that the cells classified as FD1b represent an individually identifiable neuron. In previous studies it has been shown that individual FD cells with different anatomical features could not be distinguished unambiguously on the basis of the tested functional properties (Egelhaaf, 1985b; Gauck and Borst, 1999). The term "FD1b cell" will therefore be used in the following to refer to cells with common functional properties and is not meant to imply that the cells distinguished in this way represent an individually identifiable neuron. In contrast, HS cells can be individually identified on the basis of anatomical and functional criteria (Hausen, 1982a). If not stained, HS cells were identified according to their physiological response properties: (1) Preferred direction of pattern motion in the ipsilateral part of the visual field: front-to-back. (2) Response mode: graded membrane potential changes with superimposed spike-like depolarizations. (3) No graded membrane potential changes and no IPSPs during front-to-back motion in the contralateral part of the visual field. There exist three individually identifiable HS cells that differ in the vertical position of their receptive field (Hausen, 1982a). The data of the recorded HS cells were pooled, because no systematic differences were found in the responses of the different HS cell types under the stimulus condition used in the present study.

Visual stimuli. The visual stimuli were presented in a cylindric lightemitting diode (LED) arena that had a diameter of $37 \mathrm{~cm}$ and a height of $15 \mathrm{~cm}$. It consisted of a total of 480 columns of LEDs. Each column could be switched on or off independently. The horizontal spatial resolution thus amounted to $0.75^{\circ}$. A more detailed description of the arena is given elsewhere (Kimmerle et al., 2000). The fly was positioned in the center of the arena. To obtain access to the fly's brain with the electrodes, the arena was opened $\left(108^{\circ}\right)$ in the rear. A net of thin wire was placed in front of the LEDs to shield the recording site from electrical fields. As a convention, azimuthal positions will be given with respect to the longitudinal body axis of the fly, positions $<0^{\circ}$ denoting the part of the arena in the contralateral (left), and positions $>0^{\circ}$ the part of the arena in the ipsilateral (right) half of the visual field. Vertical square wave gratings with a spatial wavelength of $7.5^{\circ}$ were generated at a frame rate of $200 \mathrm{~Hz}$

For identification of FD1b cells different motion stimuli were used. The identification procedure consisted of three steps, the third step being performed only with part of the cells. (1) The azimuthal position of the maximal sensitivity of the cells was determined in part of the experiments with a handheld probe. For 10 cells the spatial sensitivity distribution along the horizontal extent of the receptive field of the cells was determined quantitatively by horizontally oscillating a $15^{\circ}$-wide segment of the arena grating ("object") alternately around 11 different horizontal positions: $-105^{\circ},-75^{\circ},-45^{\circ},-15^{\circ},+0^{\circ},+15^{\circ},+30^{\circ},+45^{\circ},+60^{\circ},+90^{\circ}$, and $+105^{\circ}$. (2) The overall organization of the inhibitory input was analyzed quantitatively in all cells $(n=17)$ by horizontally oscillating the following parts of the arena grating alternately: an object (oscillating around a position of $+15^{\circ}$ ), the whole grating, the grating in the ipsilateral half of the visua field, an object at $15^{\circ}$ plus the part of the contralateral grating more lateral than $-24^{\circ}$ (oscillating in phase), an object at $15^{\circ}$ plus the part of the contralateral grating more lateral than $-24^{\circ}$ (oscillating in counterphase). (3) A detailed analysis of the spatial distribution of the inhibitory input was performed for part of the cells $(n=11)$. An object oscillating horizontally around a position of $+15^{\circ}$ was presented either alone or together with a second $15^{\circ}$-wide object oscillating alternately around eight different positions: $-105^{\circ},-75^{\circ},-45^{\circ},-15^{\circ},+37^{\circ},+60^{\circ},+90^{\circ}$, and $+105^{\circ}$. If the second object was on the contralateral side, the oscillations were either in phase or in counterphase. If it moved in the ipsilateral visual field, the second object was oscillated only in phase with the other object. In all three steps of the identification protocol the different stimuli were presented for $2 \mathrm{sec}$ in pseudorandom order and were separated by a $1 \mathrm{sec}$ pause. Five sequences of all stimuli were presented successively. Oscillatory motion was always sinusoidal with an amplitude of $\pm 10^{\circ}$ and a frequency of $1 \mathrm{~Hz}$. Peak velocities thus amounted to $\pm 57^{\circ} / \mathrm{sec}$.

The major goal of this study was to characterize the responses of FD1b and HS cells in behavioral situations during object fixation. Because it is hardly possible to record from these neurons during flight, optic flow stimuli were created in previous behavioral experiments in the LED arena with tethered flies under closed-loop conditions (Kimmerle et al., 2000). The optic flow stimuli generated in this way were replayed in the present study while recording from the neurons. In the behavioral experiments a background grating (wavelength, $7.5^{\circ}$ ) was superimposed by a $15^{\circ}$-wide identically textured object (see Fig. $2 a$ ). As in free flight, turning responses of the fly to either side elicited rotational motion of both object and background in the opposite direction. Translational flight was simulated by adding to the rotational motion front-to-back (translational) motion in both parts of the fly's visual field. Different distances of object and background were simulated by varying their translational velocities, thus introducing relative motion between object and background. Translational velocities were constant along the azimuth. It should be noted that these motion sequences, although they were generated by the actions and reactions of a behaving fly, are still only a first approximation to situations encountered by freely moving animals. For instance, in free flight the translational retinal velocity of an object (and of the background) depends on distance and on azimuthal position: it is zero in the heading direction (i.e., right in front of the fly) and increases toward more lateral positions. The interpretation that the present experiments simulate flight situations during which the fly encounters an object at a certain distance and then tries to keep heading toward it is thus not valid in a strict sense. Despite these qualifications the optic flow generated in this way had spatial and temporal properties characteristic of a behavioral fixation situation and the fly did fixate the object for often extended periods of time.

To investigate the influence of object and background motion on the neuronal responses three of the motion traces generated by the fly in the behavioral experiments under different conditions were selected and replayed while recording the activity of FD1b and HS cells. In the respective behavioral experiments the simulated distances of object and background (their translational velocities) were as follows: first replay condition: close object $\left(60^{\circ} / \mathrm{sec}\right)$, infinitely distant background $\left(0^{\circ} / \mathrm{sec}\right)$; second replay condition: close object $\left(60^{\circ} / \mathrm{sec}\right)$, distant background $\left(15^{\circ} / \mathrm{sec}\right)$; third replay condition: very close object $\left(240^{\circ} / \mathrm{sec}\right)$, distant background $\left(15^{\circ} / \mathrm{sec}\right)$. The three motion traces were presented in their original and in two modified versions. In the original motion traces the object appeared $5 \mathrm{sec}$ after the onset of flight simulation at an azimuthal position of $0^{\circ}$. The first modification consisted in stopping background motion at the instance when the object appeared. In the second modification the object was not displayed at all, whereas the background continued to move as in the original motion trace, and the area where the object was in the original trace was filled by background motion. In addition, the resulting set of motion traces was mirrored with respect to the sagittal plane of the fly. The response to a mirrored stimulus was considered to be equivalent to the response of the respective cell in the contralateral optic lobe to the non-mirrored stimulus. The complete set of 18 replay stimuli (three conditions $X$ three versions $\times$ two mirror symmetric traces) was presented in pseudorandom order. Each individual stimulus lasted $10 \mathrm{sec}$. The whole set was presented 10 times to six FD1b cells and nine times to five HS cells. The respective data are shown in Figures $2-7$.

To investigate the influence of background distance on the cellular responses another set of motion traces created in three behavioral experiments was selected and replayed. The translational velocities of object (background) in each of the three flight simulations amounted to $60^{\circ} / \mathrm{sec}$ $\left(15^{\circ} / \mathrm{sec}\right)$ (i.e., close object, distant background). Each of these three motion traces was presented in its original and in three modified versions. The modifications were: (1) Background motion was stopped completely. (2) The translational component of background motion was removed, or (3) increased to $30^{\circ} / \mathrm{sec}$, whereas the rotational component was left unaltered. The modifications applied to the entire motion trace. Object motion was always as in the original replay. The resulting stimuli were also mirrored with respect to the sagittal plane of the fly and presented in pseudorandom order. Each individual stimulus lasted $10 \mathrm{sec}$. The whole set of 24 replay stimuli (from three behavioral experiments $\times$ four versions $\times$ two mirror symmetric traces) was presented 14 times to five FD1b cells and 10 times to four HS cells. The respective data are shown in Figure 8.

Data analysis. Peristimulus time histograms (PSTHs) of the spike activity were calculated for each of the recorded FD1b cells with a temporal resolution of $1 \mathrm{msec}$, subsequently averaged over cells and smoothed with a rectangular filter that had a width of 51 msec. Likewise, the membrane potential traces were first averaged for each HS cell, subsequently averaged over cells, and smoothed with the same filter. The resting potential of all nine HS cells of which the responses to replay stimuli were recorded varied 
Figure 1. Characterization of FD1b cells based on their spatial integration properties. $a$, Spatial sensitivity distribution along the horizontal extent of the eyes. Time-averaged responses to sinusoidal oscillation of a $15^{\circ}$-wide object at 11 azimuthal positions. Spike rates were determined during clockwise motion (ipsilateral front-to-back, contralateral back-to-front). After subtracting the spontaneous activity the responses were normalized to the maximal response. This procedure was applied to each cell. The average spontaneous activity was 19 spikes/sec; the average maximal activity amounted to $87 \mathrm{spikes} / \mathrm{sec}$. The figure shows the mean and SEM obtained from 10 cells. Dashed line represents the frontal position in the visual field. $b$, Inhibition during large-field motion. Plotted is the ratio of the responses to different types of oscillatory large-field motion $\left(R_{\mathrm{LF}}\right)$ and to motion of a $15^{\circ}$-wide object oscillating about an ipsilateral azimuthal position of $15^{\circ}\left(R_{\mathrm{SF}}\right)$. The large-field stimuli were (1) the entire (binocular) grating, (2) the grating in the ipsilateral part of the visual field, (3) part of the contralateral grating oscillating in phase with the object, and (4) part of the contralateral grating oscillating in counterphase with the object. The responses were determined during ipsilateral front-to-back motion. Schemat-
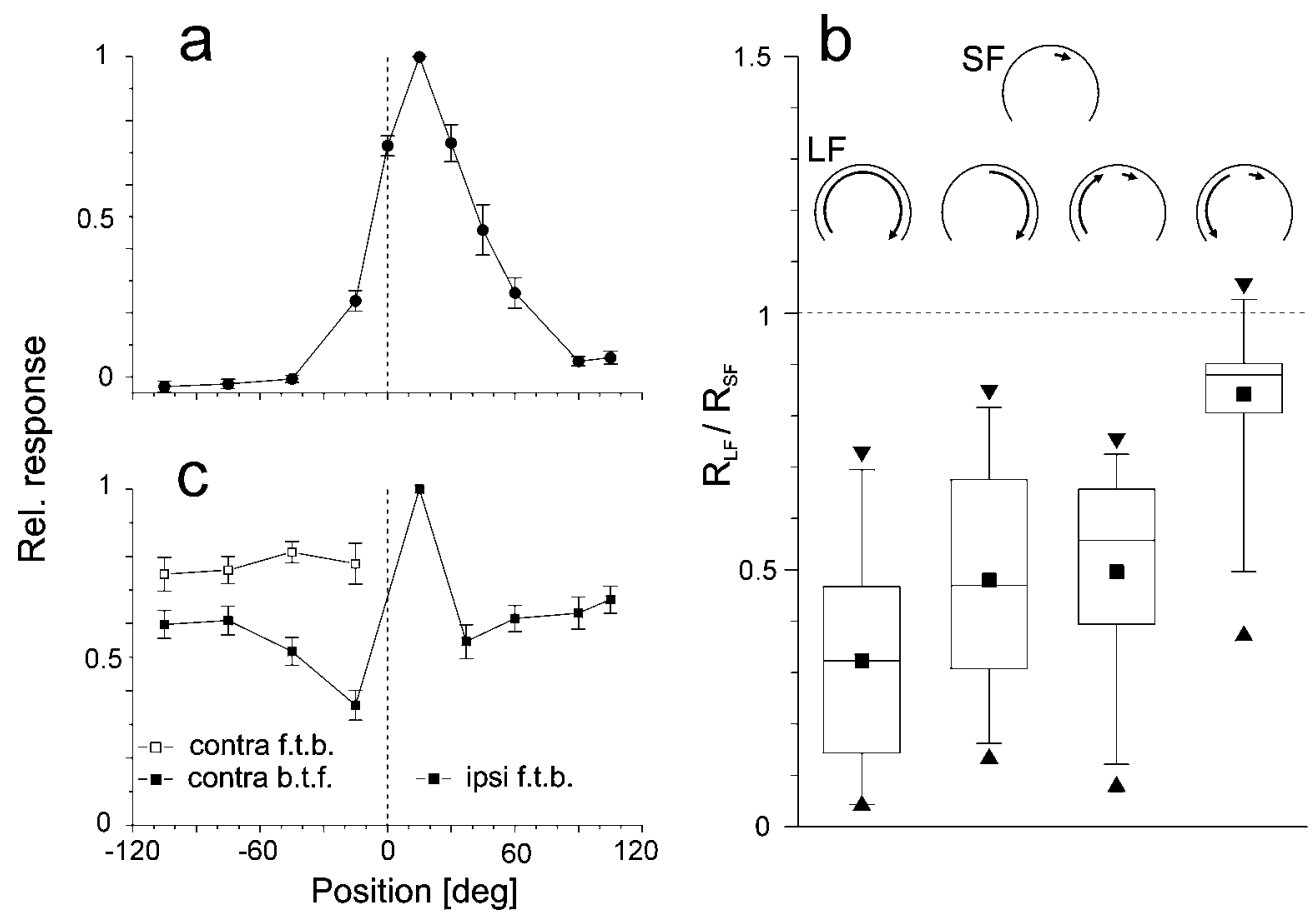

ics (top) represent motion conditions in the arena (as viewed from above) during SF and LF motion. Box charts show the median, the quartiles, as well as the 5th and 95th percentiles of the respective distribution. Extrema are indicated by triangles, means are indicated by squares. Values below the dashed line indicate inhibition by large-field motion. $n=17$ cells. $c$, Spatial sensitivity distribution of the inhibitory input. Responses of single cells were determined as average firing rates during simultaneous oscillation of two $15^{\circ}$-wide objects. One object oscillated in the center of the excitatory receptive field around an azimuth of $+15^{\circ}$, the other one around variable positions either in phase (filled symbols) or in counter phase (open symbols). The time-averaged responses were determined during front-to-back motion of the object at $+15^{\circ}$. Relative response strengths were obtained as described in a. f.t.b., Front-to-back; b.t.f., back-to-front. $n=11$ cells; Error bars indicate SEM.

in the range from -35 to $-51 \mathrm{mV}$ (average, $-43 \mathrm{mV}$ ). The spontaneous activity of all $11 \mathrm{FD} 1 \mathrm{~b}$ cells of which the responses to replay stimuli were recorded varied in the range from $1 \mathrm{spike} / \mathrm{sec}$ to $72 \mathrm{spikes} / \mathrm{sec}$ (average, 16 spikes/sec).

Behavior is controlled by the neurons in both brain hemispheres. Thus, the responses of FD1b and HS cells ipsilateral as well as contralateral to object motion have to be considered. The most simple assumption about how the signals of both brain halves interact is that there are no nonlinear interactions down to the level of the motor output. In this case, because of the bilateral symmetry of the flight motor, the turning responses could be explained on the basis of the differences between the neuronal signals of both brain halves. It has been suggested that in the fly in the context of course stabilization by the optomotor system the signals of both brain hemispheres are subtracted (Götz, 1975). Because no model of bilateral interaction has been proposed so far for object fixation, the simplest mechanism, i.e., subtraction, was chosen as a working hypothesis also for the interaction of the signals originating from FD1b cells.

The average responses to object and to background motion in the preferred direction of the cells (see Fig. 5) were determined after correcting for response phase shifts. The latter were determined by crosscorrelating the responses with the corresponding velocity trace. The resulting phase shifts for the different replay conditions ranged from $0 \mathrm{msec}$ (very close object) to $60 \mathrm{msec}$ (infinitely distant background).

Responses to the original replay stimuli during the fixation period were compared with the responses to the modified replay motion traces during the corresponding time interval (see Figs. $6-8$ ). The fixation period was defined to end when the object had reached a lateral position of $\pm 60^{\circ}$. This was the case after $4.67 \mathrm{sec}$ (see Fig. 7, first replay condition), $470 \mathrm{msec}$ (see Fig. 7, third replay condition), $3.95 \mathrm{sec}$ (see Fig. 8, first motion trace). In the remaining replay motion traces the fixation period lasted until the end of the trial. The responses were compared in two ways: (1) The average response amplitudes were determined. (2) Cross-correlograms (CCGs) of the responses were calculated after subtracting the mean response amplitude. The CCGs were normalized to the autocorrelation of the response to the original replay version. Because of the discrete response mode (spiking) of the FD1b cells all CCGs were calculated after averaging the response traces over cells and smoothing (see above). The time interval used to compute CCGs started $250 \mathrm{msec}$ after the object appeared and ended $250 \mathrm{msec}$ before the end of the fixation period.

\section{RESULTS}

\section{Spatial integration properties of the FD1b cell}

FD1b cells are excited by front-to-back motion of small objects in the ipsilateral visual field and are inhibited by motion in the opposite direction. The excitatory receptive fields of the FD1b cells as recorded in the present study were centered around an azimuthal position of $+15^{\circ}$. Their frontal margins were determined to lie between $-15^{\circ}$ and $-45^{\circ}$ and their lateral margins to lie between $+60^{\circ}$ and $+90^{\circ}$ (Fig. $1 a$ ). The distinguishing feature of all FD cells is that they respond stronger to motion of small objects than to large-field motion (Egelhaaf, 1985b). This was also the case for FD1b cells (Fig. 1b). Inhibition of the FD1b cell was strongest, when rotational large-field motion around the fly's vertical body axis was presented binocularly and weaker when large-field motion was restricted to the ipsilateral side of the visual field. Based on their preferred direction of motion (front-to-back) and the location of their excitatory receptive field (centered at $+15^{\circ}$ ) the FD1b cells could be classified as FD1 according to the original classification system for FD cells (Egelhaaf, 1985b). However, unlike FD1 cells, they were inhibited by contralateral motion not only in one but in both directions and were therefore named FD1b (Fig. 1b; Kimmerle and Egelhaaf, 2000). Inhibition was stronger during contralateral back-to-front motion than during front-to-back motion (Fig. 1b). Contralateral front-to-back motion reduced the activity of the FD1b cell to simultaneous motion of an object at $+15^{\circ}$ virtually independent of the stimulus position in the contralateral visual field (Fig. 1c). In contrast, inhibition by contralateral backto-front motion was stronger in the frontal part of the visual field.

\section{Cellular responses to behaviorally generated optic flow}

The results shown in Figure 1 indicate that the selectivity of FD1b cells for moving objects is attributable to an inhibitory input reducing their firing rate during background motion. The question thus arises how well these cells signal object motion in behavioral situations in which the optic flow is continuously changed by the animal's flight behavior and in which the eyes usually are confronted with simultaneous object and background motion. Moreover, how do the responses of FD1b cells compare with responses of cells that are not inhibited by large-field motion, such as the HS cells? 


\section{$1^{\text {st }}$ behavioral condition: object close background infin. distant}

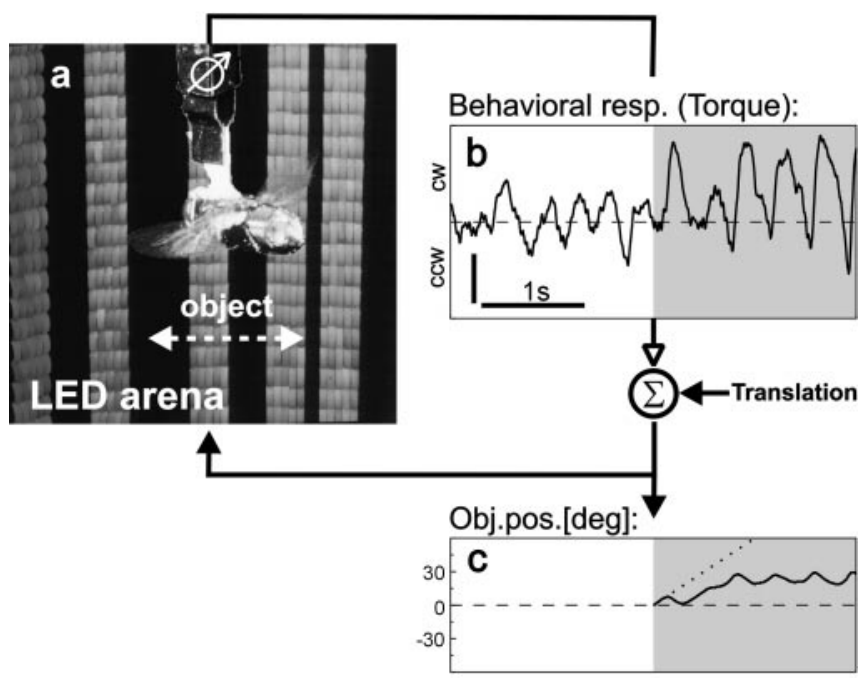

\section{$1^{\text {st }}$ replay condition:}

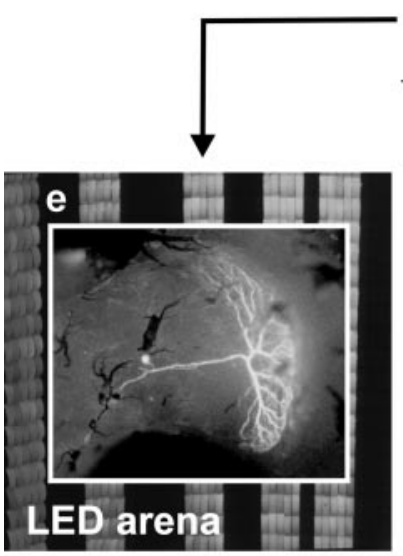

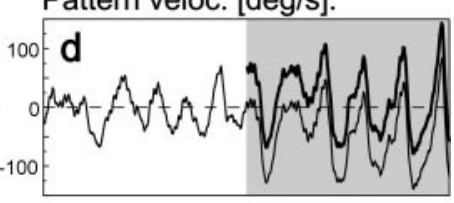

FD1b-cell resp. [spikes/s]:

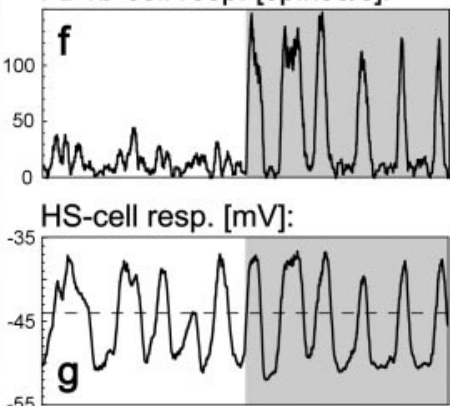

Time

Figure 2. Object fixation behavior and replay of behaviorally generated stimuli. $a$, Fly in the flight simulator. White arrow indicates the horizontal extent of the object. The remaining part of the grating is background. $b$, Torque response of the fly before (white area) and after (shaded area) object appearance. The torque responses were coupled to the rotational component of object and background motion. By adding translational motion the simulated distances of object and background could be varied. In the example shown here a close object was simulated in front of an infinitely distant background ( $1^{\text {st }}$ replay condition; see Materials and Methods). Dashed line indicates zero torque (straight flight). Vertical scale bar: $5 \times$ $10^{-7} \mathrm{Nm} ; c c w$, counterclockwise; $c w$, clockwise, $c$, Object position as resulting from the fly's fixation behavior (solid line) and as it would have been under open-loop conditions (dotted line). $d$, Velocity of background (thin line) and object (thick line) resulting from the fly's torque response as shown in b.e. Behaviorally generated stimuli were replayed while recording the activity of FD1b and HS cells in the right optic lobe (inset shows, for illustrative purposes, a Lucifer yellow-stained FD cell in Calliphora that was excited by front-to-back motion in the frontal part of the visual field; because the spatial properties of its inhibitory input were not tested systematically, it cannot be classified unambiguously as FD1b cell). $f$, g, Time course of the spike rate of FD1b cells ( $f, n=6$ cells) and the membrane potential of HS cells ( $g, n=5$ cells) during replay. The same time interval is shown in $b-d$, $f$, and $g$.

Optic flow stimuli generated by the fly in a flight simulator during object fixation (Kimmerle et al., 2000) were replayed while recording the activity of FD1b and HS cells (Fig. 2). In the flight simulator (Fig. $2 a$ ) the yaw torque of the fly was continuously

\section{a $2^{\text {nd }}$ replay condition: Object close \\ Backgr. distant}

\section{b $3^{\text {rd }}$ replay condition: Object very close Backgr. distant}
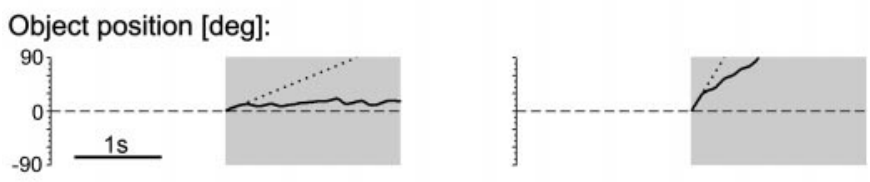

Pattern velocity [deg/s]:
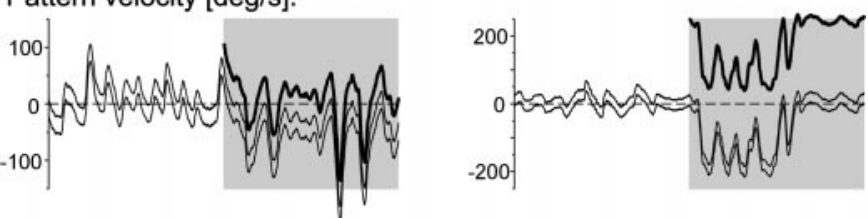

FD1b-response [spikes/s]
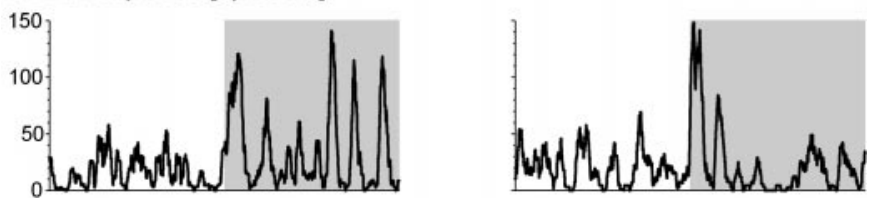

HS-response [mV]:

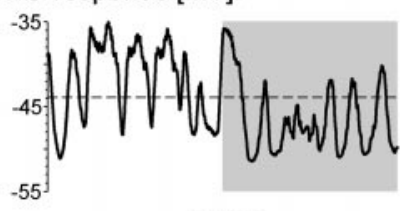

Time

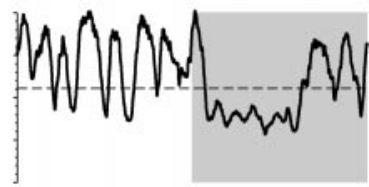

Time

Figure 3. Responses of FD1b and HS cells in different object fixation situations. $a, b$, Second and third replay condition (see Materials and Methods). Top row, Object position as resulting from the fly's fixation behavior (solid line) and as it would have been under open-loop conditions (dotted line) in the respective flight simulation. Second row from top, Velocity of the background in the right/left visual hemifield (top, bottom thin line) and of the object (thick line) as resulting from the fly's turning behavior in the respective flight simulation. Different background velocities in both visual hemifields are attributable to simulated translation and the background's finite distance. Bottom rows, Time course of the spike rate of FD1b cells (third row from top, mean from $n=6$ cells) and the membrane potential of HS cells (bottom row, mean from $n=5$ cells) during replay of the motion stimuli generated in the respective flight simulation. The same time interval is shown in all plots.

measured (Fig. $2 b$ ) and directly coupled to the velocity of object and background. As in free flight, a clockwise torque resulted in counterclockwise pattern rotation around the vertical axis of the fly and vice versa. To simulate a situation in which the fly passes a close object in front of an infinitely distant background, a constant front-to-back velocity ("translational motion") was added to the object motion but not to the background motion. During an initial period of background motion alone the fly tried to stabilize the retinal image, as indicated by torque fluctuations around the zero level (Fig. 2b, white area; Heisenberg and Wolf, 1988; Warzecha and Egelhaaf, 1996). Then the object appeared in front of the fly, and the fly tried to turn toward it as can be inferred from the shift of the average turning strength to positive values (Fig. $2 b$, shaded area). As a consequence of this response the object could be fixated by the fly in the frontolateral part of the visual field (Fig. 2c). The continuous torque fluctuations produced by the fly led to pronounced velocity fluctuations of object and background (Fig. $2 d$ ).

The motion traces generated in this way were replayed (Fig. 2e) while recording the responses of FD1b and of HS cells. Before the object appeared in the visual field, the FD1b cells fired only weakly (Fig. 2f). However, after appearance of an object strong responses were elicited during object motion in the preferred direction of the FD1b cell. The response modulated with the velocity of the object. In contrast, the HS cells strongly responded to background rotation before the object appeared (Fig. $2 g$ ). The changes in the membrane potential mainly followed the time course of the background ve- 
FD1b - response [ spikes/s ]:

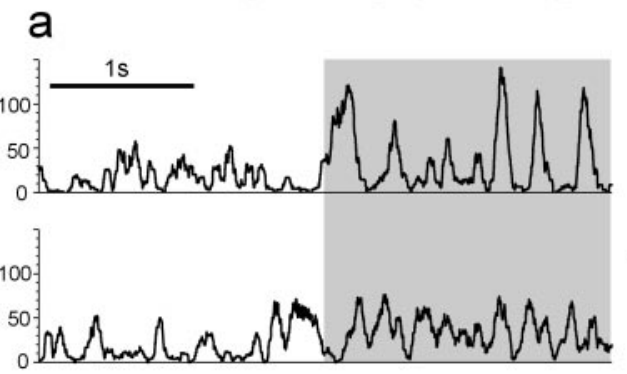

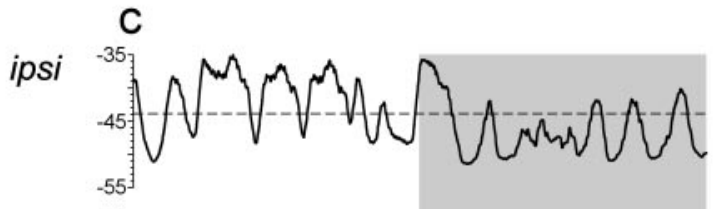

contra

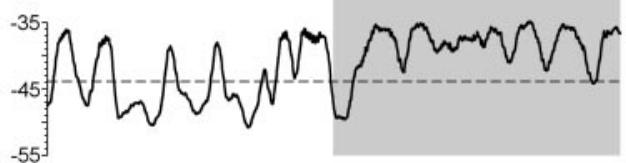

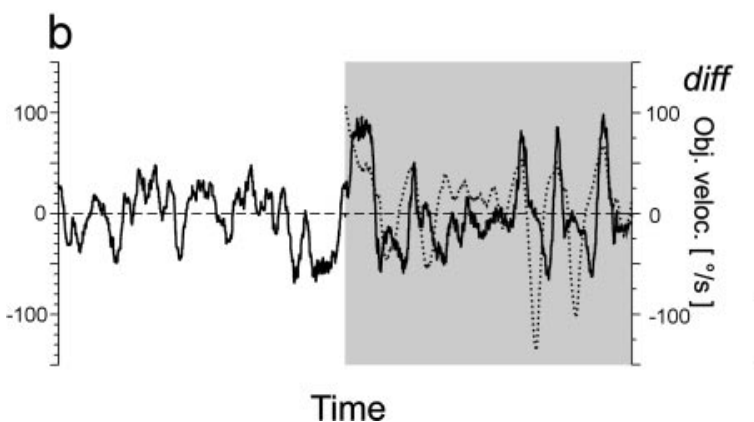

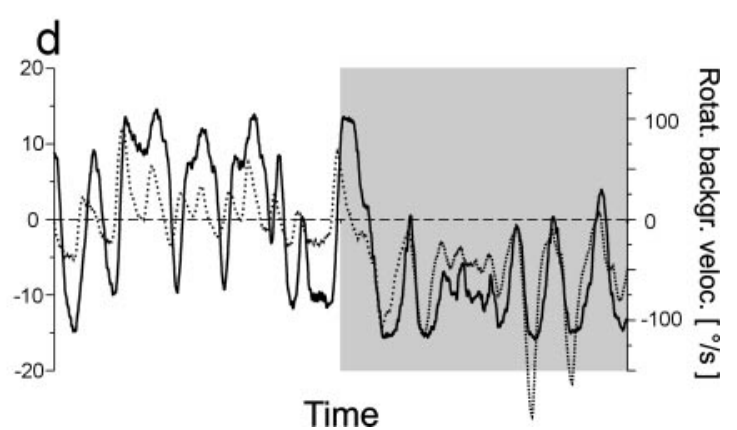

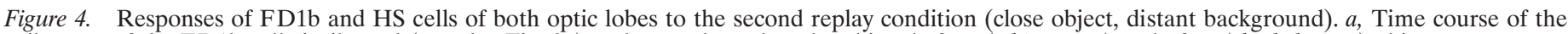

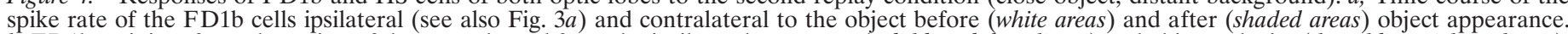

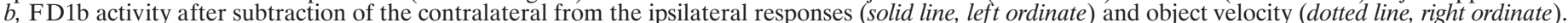

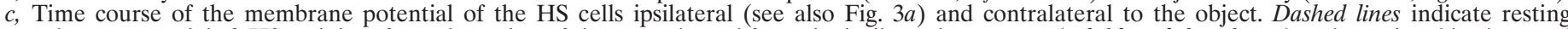

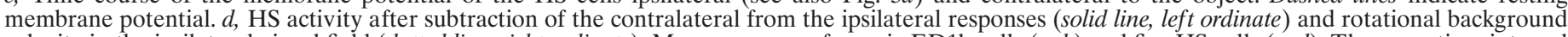

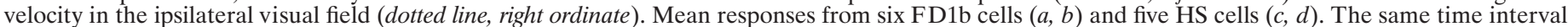
is shown in all plots.

locity. After appearance of the object the membrane potential fluctuations continued to follow the rotational velocity of object and background without obvious changes.

Further fixation trials were chosen for replay (Fig. 3). The motion stimuli used in the second replay condition originated from a behavioral situation in which the background was simulated to be distant and the object to be close (Fig. $3 a$ ). After the object was introduced, it was fixated in the frontal part of the right visual field (Fig. 3a, top panel). As a consequence, the velocity of the object was fluctuating around zero, whereas the background in both parts of the visual field was drifting in the direction opposite to the turning direction (Fig. $3 a$, second panel from top). As in the first replay condition, the FD1b cell fired only weakly during background motion alone. Stronger modulations of the firing rate were measured during object motion (Fig. $3 a$, third panel from top). The membrane potential of the HS cells was strongly modulated by background motion before the object appeared (Fig. 3a, bottom panel). Because the background was translating from front to back, corresponding to the preferred direction of the HS cells, an average depolarization of the HS cells was observed. During object fixation, the HS cells were, on average, hyperpolarized because of the background drifting in the opposite of the preferred direction of the cells.

The motion traces used in the third replay condition were generated in a behavioral experiment in which the object was simulated to be very close and the background to be distant (Fig. 3b). The fly could not fixate the object over an extended period of time (Fig. 3b, top panel). However, several strong turns shortly after the start of object motion compensated the object's translational velocity to some extent for a brief period of time (Fig. 3b, second panel from top). The FD1b cell responded to the appearance of the fast object with a strong activity increase (Fig. 3b, third panel from top). Because the object moved out of the receptive field of the FD1b cell very quickly, the response was only short. The HS cells were on average depolarized before the object appeared because of the translating background (Fig. $3 b$, bottom panel). During the attempt of the fly to fixate the object, the concurrent counterrotation of the background led to a hyperpolarization of the HS cells.
In summary, FD1b cells fired weakly as long as no object was present, whereas the membrane potential of HS cells strongly modulated with the background velocity. FD1b cells started responding strongly after the object appeared in their receptive field. In contrast, the response of HS cells during object fixation seemed to be less strongly influenced by object motion and more strongly influenced by background motion.

\section{Responses of cells contralateral to the object}

In all experiments described so far, the object was moving in the visual field ipsilateral to the recorded cells. Because visually guided behavior is mediated by neurons in both halves of the brain, one has also to take into account the responses of the respective cells contralateral to object motion. In the present experiments, the responses of FD1b and $\mathrm{HS}$ cells contralateral to object motion were inferred from the responses of the recorded cells to the mirror symmetric versions of the stimuli. To interpret the responses of neurons in both halves of the brain with respect to their potential significance in visually guided orientation behavior, one needs an assumption about how their signals interact on the way to the motor output. As a working hypothesis, the signals originating from the ipsilateral and the contralateral neurons were supposed to be subtracted (for an explanation, see Materials and Methods).

As expected, unlike ipsilateral FD1b cells contralateral FD1b cells did not respond with a strong increase in firing rate modulation after object appearance in the second replay condition (Fig. $4 a$ ). However, a slight increase of the average firing rate as compared to the period before object appearance was observed (Fig. $4 a$, bottom curve). This increase can be explained by the fact that object fixation was accompanied by background rotation in the preferred direction of the contralateral FD1b cells.

Subtraction of the ipsilateral and contralateral FD1b responses led to an attenuation of the response increase as was recorded in ipsilateral FD1b cells after object appearance (Fig. 4b). The firing rate modulations followed the changes in object velocity rather closely. However, fast negative velocity transients of the object are only partly reflected in the response trace. This can be explained by the limited dynamical response range of the ipsilateral FD1b cell 


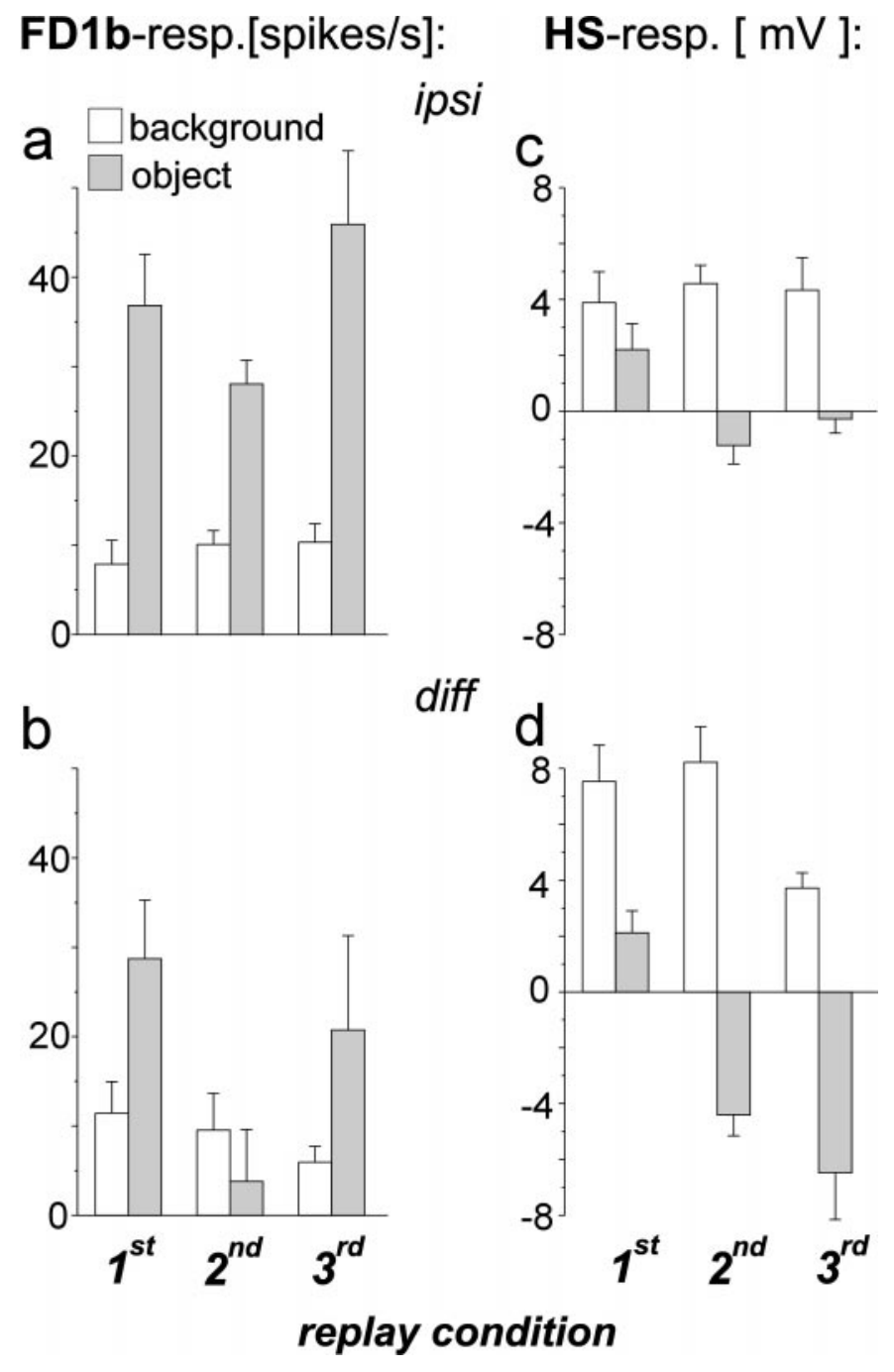

Figure 5. Specificity of FD1b and HS cells for object and background motion. Average response amplitudes during periods in which the ipsilateral background moved in the preferred direction of both cell types in the absence of an object (white columns) and during object motion in the preferred direction (shaded columns). $a$, Responses of FD1b cells ipsilateral to the object as obtained after subtraction of the spontaneous activity (average, 9 spikes/sec). $b$, Difference between the responses of the FD1b cells of both optic lobes. $c$, Responses of the HS cells ipsilateral to the object as obtained after subtraction of the resting potential (average, $-44 \mathrm{mV}$ ). $d$, Difference between the responses of the HS cells of both optic lobes. $n=$ 6 cells $(a, b) ; n=5$ cells $(c, d)$. Error bars indicate SEM.

during motion in the anti-preferred direction, which is attributable to the relatively low resting activity of the cell. The responses of ipsilateral and contralateral HS cells to the same replay appeared to be almost mirror-symmetrical, both before and after object appearance (Fig. 4c). As a consequence, after subtraction the signal had a very similar time course as the HS responses of each optic lobe when regarded separately (Fig. 4d). The HS response followed the changes in background velocity very closely. In the following quantitative analyses the responses obtained after subtracting the cellular signals of both halves of the brain will be considered in addition to the responses of the cells ipsilateral to the object.

\section{Specificity of the FD1b cell and HS cells for object and background motion}

How strong are the responses of both cell types to an object moving in front of its background during fixation as compared to background motion alone? The ipsilateral FD1b cells responded more strongly to object than to background motion in the preferred direction, irrespective of the replay condition (Fig. $5 a$ ). When the responses of the FD1b cells of both optic lobes were subtracted, the differences between the firing rates during object and background motion became smaller (Fig. $5 b$ ). The difference signal of the two heterolateral FD1b cells was thus less specific for preferred direction object motion than the response of the ipsilateral FD1b cell alone.

The HS cells became more depolarized during background motion than during object motion in the preferred direction (Fig. 5c). In two replay conditions the HS cells were hyperpolarized during object motion in the preferred direction, which can be explained by the concurrent motion of the background in the opposite direction. The differences between the responses to background motion and object motion became more pronounced when the signals of the ipsilateral and contralateral HS cells were subtracted (Fig. $5 d$ ). The HS cells can thus be concluded to respond rather specifically to background motion when confronted with optic flow as generated in a behavioral situation. Subtraction of the signals from both optic lobes is suited to increase the specificity of the HS cells for rotational background motion around the fly's vertical body axis.

\section{Influence of object and background motion during object fixation}

Each of the motion sequences used for replay was modified in two ways to compare the influence of object and of background motion on the responses of FD1b and the HS cells during object fixation. This approach is illustrated in Figure 6 for the second replay condition (close object, distant background). In an "only object" version background motion was stopped when the object appeared (Fig. 6a, top panel). The object moved as in the original motion trace. In an "only background" version (Fig. 6a, bottom panel) no object was displayed, whereas background motion continued in the same way as in the original motion trace. In the present example, the modulations of the firing rate of FD1b cells in response to object motion did not seem to be affected in a conspicuous way by the absence of background motion (Fig. 6b, compare top and middle response traces). This notion is supported by the high correlation of both responses (Fig. $6 b$, peak at time 0 in upper CCG). If no object was displayed, the FD1b cells almost ceased firing because of background rotation in the opposite of the preferred direction of the cells (Fig. 6b, bottom response trace). Accordingly, the correlation between the responses to original and only background replay was weak (Fig. $6 b$, lower $C C G$ ). The HS cells were, on average, less hyperpolarized during presentation of the only object version than during presentation of the original motion trace (Fig. $6 c$, compare top and middle response traces). In contrast to the effect of this modification on the average potential, the time course of the membrane potential modulations was similar during presentation of the original and modified motion traces, as indicated by the strong correlation (Fig. $6 c$, upper $C C G$ ). When presenting the only background version, the membrane potential modulations of the HS cells were weaker than during presentation of the original motion traces (Fig. $6 c$, compare bottom and middle response traces) and accordingly the correlation was comparatively weak (Fig. $6 c$, lower $C C G$ ).

Thus, whereas the responses of FD1b cells were only weakly influenced by background motion but strongly influenced by object motion, the responses of the HS cells depended in a more complex way on object and background motion: removing background motion mainly affected the average membrane potential, whereas removing the object had a stronger influence on the modulations of the membrane potential.

The influence of either modification on the cellular responses to the different replay conditions was quantified in terms of the peak of the respective CCG and in terms of the changes in the average response rate (Fig. 7). The average responses of ipsilateral FD1b cells hardly changed when the only object versions of the first two replay conditions were presented (Fig. 7a, filled symbols). In contrast, they decreased during presentation of the only background version (Fig. 7a, open symbols). Hence, the activity of FD1b cells in these situations was almost exclusively determined by object motion and independent of background motion. The only object 


\section{a Pattern velocities [ $\%$ ]:}

\section{'Only object':}

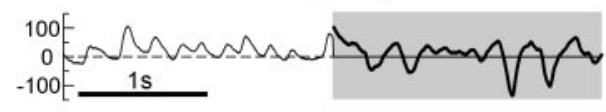

Original replay:
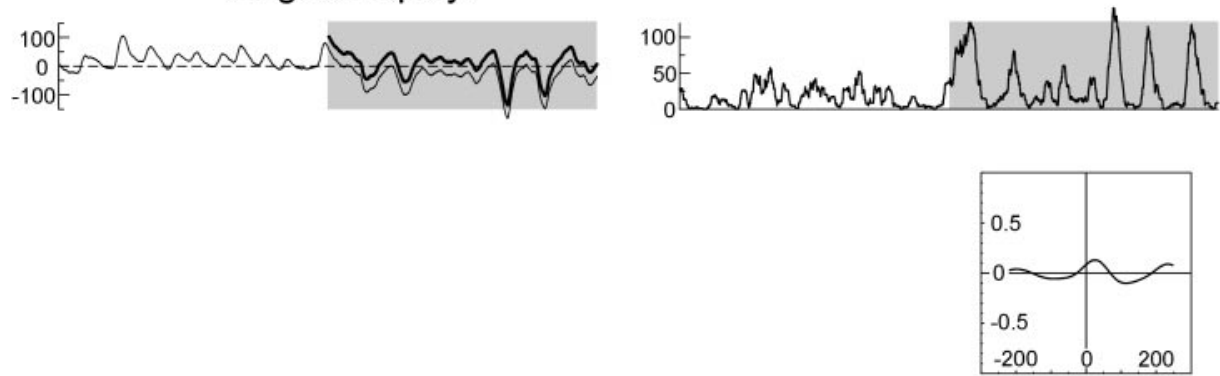

'Only backgound':

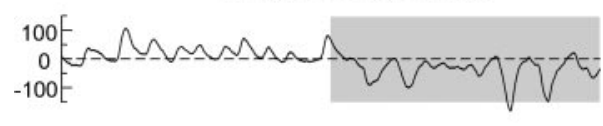

Time

\section{b FD1b - response [ spikes/s ]: C HS - response [ $\mathrm{mV}$ ]:}
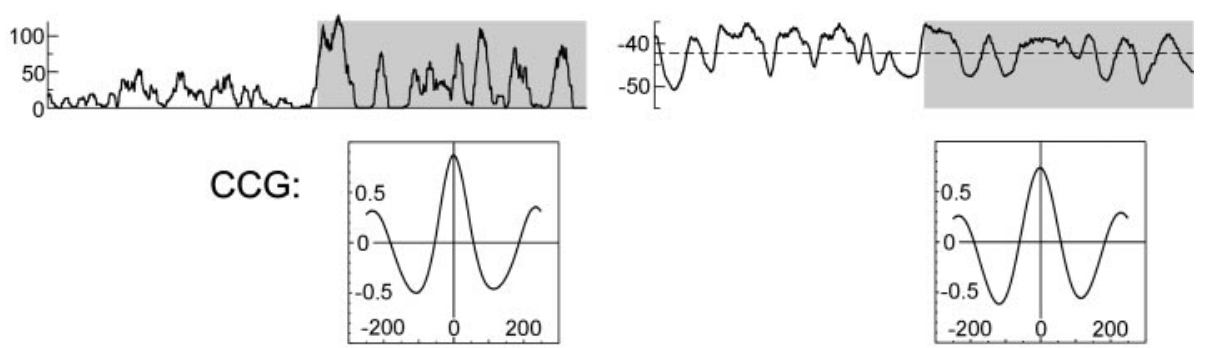

Time [ $\mathrm{ms}$ ]
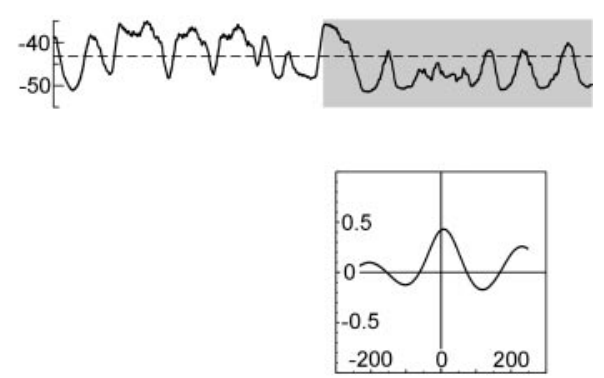

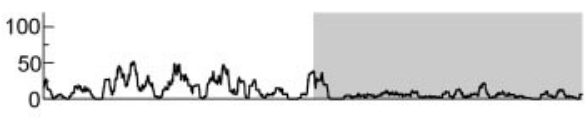

Time

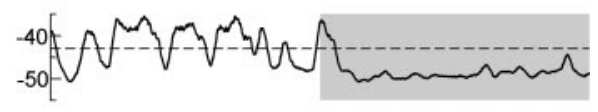

Time

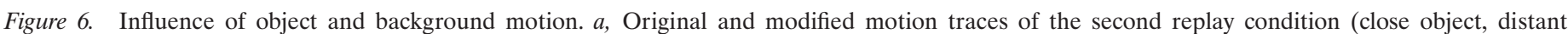

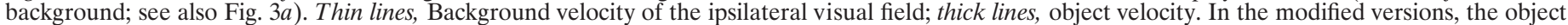

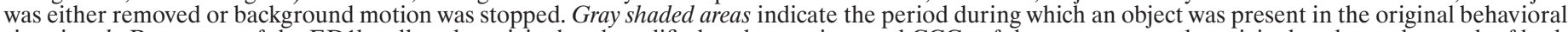

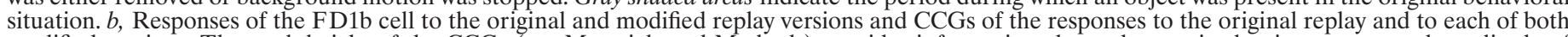

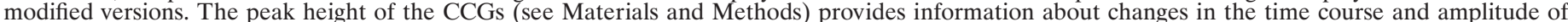

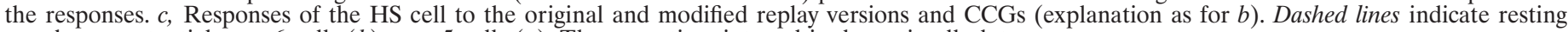
membrane potential. $n=6$ cells $(b) ; n=5$ cells $(c)$. The same time interval is shown in all plots.

version of the third replay condition increased the FD1b activity, whereas the activity in the corresponding only background version led to an activity decrease compared to the response to the original replay. After subtraction of the ipsilateral and the contralateral responses the influence of background motion, i.e., the increases in the mean firing rate caused by presentation of the only object version, became significantly more pronounced in the second replay condition $(p<0.01$, paired $t$ test; compare filled triangles in Fig. $7 a, b)$.

The temporal modulations of the firing activity of the ipsilateral FD1b cell were not much changed during presentation of the only object versions of the tested replay conditions as is indicated by the strong correlation of the respective responses as well as by direct comparison of the response traces (Fig. 7c). Thus, background motion had only very little influence on the modulation of the firing rate of the FD1b cell. The opposite was true for object motion. Almost no correlation remained between the only background and the original versions. Subtraction of the responses of the ipsilateral and contralateral FD1b cells led to the same conclusion (Fig. 7d).

The average response of the ipsilateral HS cells was influenced by both object and background motion (Fig. 7e). During presentation of the only object versions the membrane potential was raised above the level measured during presentation of the original motion traces. Presentation of the only background version resulted in a more negative average membrane potential. Subtraction of the HS signals of both optic lobes further increased the influence of background motion considerably ( $p<0.05$ for each of the replay conditions, paired $t$ test; compare filled triangles in Fig. 7e,f).

The membrane potential modulations of the ipsilateral HS cells were slightly altered during presentation of the "only object" ver- sions (Fig. 7g). More pronounced changes were obtained when object motion was removed. The influence of object motion on the membrane potential modulations of the HS cells was thus stronger than the influence of background motion. However, the differences between the modifications were not as strong as in FD1b cells. An inversion of the relative contribution of object and background motion was observed after subtraction of the responses of the HS cells in both optic lobes (Fig. $7 h$ ). In this case stopping background motion had a stronger impact on the response modulation than removing the object.

In summary, replaying optic flow experienced by a fly in three different behavioral situations of object fixation revealed that in each situation object motion was the key determinant for the activity of FD1b cells. Background motion had only little influence on the FD1b cell responses, although this cell is assumed to owe its selectivity for object motion to inhibitory input from cells sensitive for large-field motion. The responses of the HS cells depended on both object and background motion. Subtraction of the responses of both optic lobes led to an increase of the influence of background motion in both cell types.

\section{Influence of simulated background distance}

Does the distance between object and background have any influence on the responses of FD1b and HS cells to object motion? To answer this question, three fixation trials were chosen from the behavioral experiments in which the simulated distances between object, background, and fly were the same (object close, background distant). The translational component of background motion of each of the three motion traces was subsequently modified (1) by stopping background motion completely, (2) by eliminating 


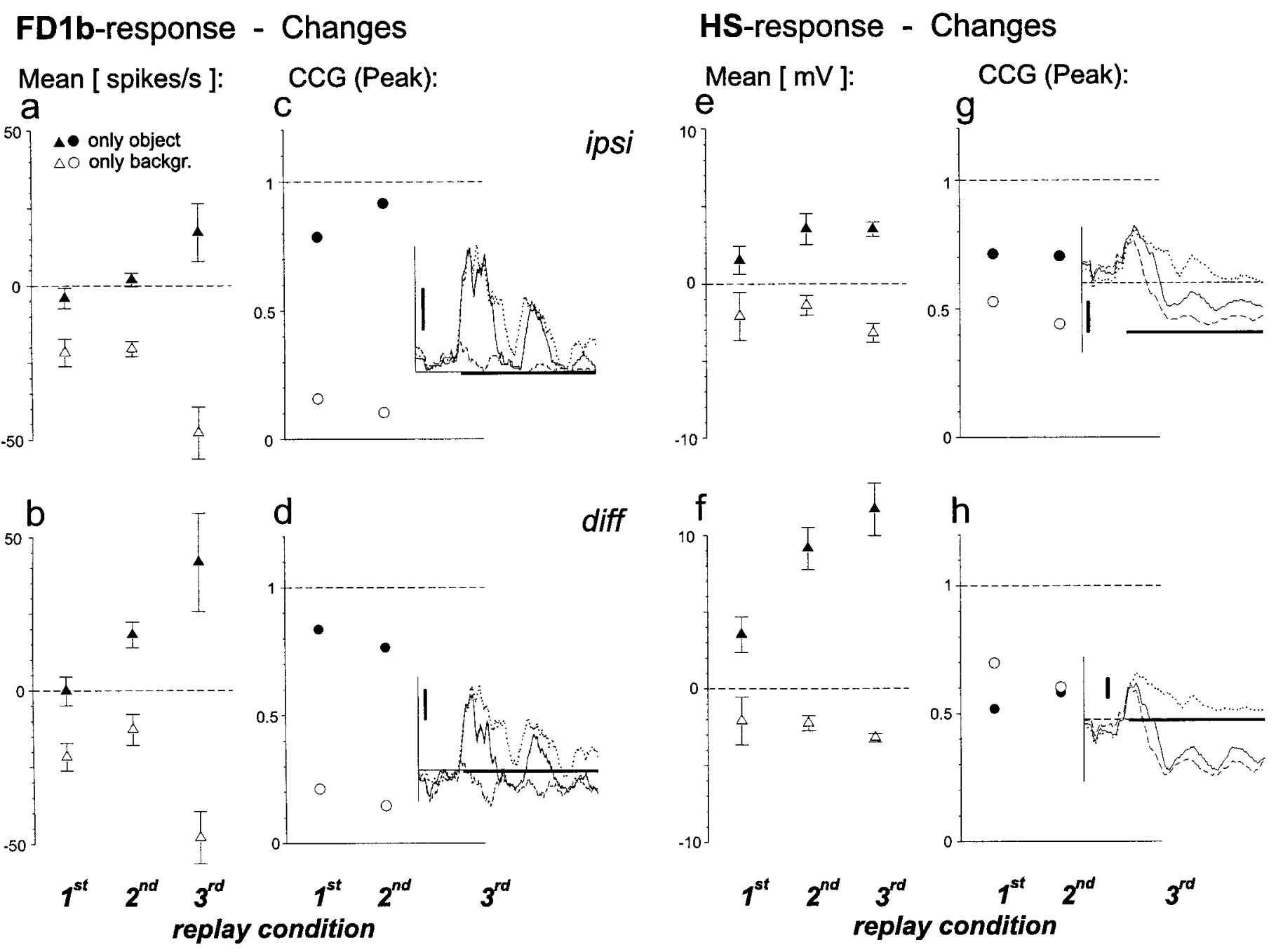

Figure 7. Quantification of the influence of object and background motion. Filled symbols, Changes caused by stopping background motion during the period of object fixation (see Materials and Methods). Open symbols, Changes caused by removing the object. $a$, Changes in the average spike rate of the FD1b cell ipsilateral to object motion. Average response in the first, second, and third original replay was 31, 28, and 57 spikes/sec, respectively. $b$, Changes in the average FD1b response after subtraction of the activity of the contralateral cell. $c$, Peaks of the CCGs between the FD1b responses to original and modified motion traces. No CCGs were calculated for the responses to the motion traces of the third replay condition caused by the short fixation period. Instead the time courses of the responses to the original (solid line), the only object (dotted line), and the only background (dashed line) versions are shown. Horizontal scale bar $(0.6 \mathrm{sec})$ indicates the appearance of the object in the respective behavioral experiment. Vertical scale bar: $50 \mathrm{spikes} / \mathrm{sec} . d$, Responses of the contralateral FD1b cell were subtracted before calculation of the CCGs. Further explanations as for $c . e$, Changes in the average membrane potential of the HS cells ipsilateral to object motion. Average response (deviation from resting potential) in the first, second, and third original replay was -0.8 , -2.7 , and $-0.2 \mathrm{mV}$, respectively. $f$, Changes in the mean HS response after subtraction of the activity of the contralateral cell. $g$, Peaks of the CCGs between the HS responses to original and modified motion traces. Straight dashed line in the inset, Resting membrane potential. Vertical scale bar, $5 \mathrm{mV}$. Further explanations as for $c$. $h$, Responses of the contralateral HS cells were subtracted before calculation of the CCGs. Further explanations as for $c$ and $g$. $n=$ 6 cells $(a-d) ; n=5$ cells $(e-h)$. Error bars indicate SEM.

the translational component of background motion, thus mimicking an infinitely distant background, or (3) by increasing the translational velocity to simulate a closer background. These changes refer to the entire trial, i.e., before and after object appearance. The average firing activity of ipsilateral FD1b cells neither changed significantly when background motion was stopped nor after increasing or decreasing the translational velocity of background motion and thus the simulated distance of the background to the fly (Fig. $8 a$, paired $t$ test). However, an influence of background translation was observed when the signals of the FD1b cells of both optic lobes were subtracted (Fig. 8b). In this case, the firing rate decreased with increasing velocity of background translation, i.e., with increasing background proximity. The modulation of the firing activity of FD1b cells was neither much affected by stopping background motion nor by changing its translational velocity. This was true for both the responses of the ipsilateral cells (Fig. $8 c$ ) as well as for the subtracted signal from both optic lobes (Fig. $8 d$ ).

The average membrane potential of ipsilateral HS cells was more positive when background motion was stopped (Fig. $8 e ; p<0.05$ for the first and second motion trace, not significant for the third motion trace; paired $t$ test). No changes were observed after eliminating the translational component of background motion or after increasing the translational velocity. The same conclusion could be drawn when the signals of the cells of both optic lobes were subtracted (Fig. $8 f$ ). The membrane potential modulations of the HS cells were somewhat attenuated by stopping background motion (Fig. 8g). This effect was increased when the signals of both optic lobes were subtracted (Fig. 8h). Changing the velocity of background translation did not much affect the membrane potential modulations of the HS cells.

In summary, stopping background motion increased the average responses and attenuated the membrane potential modulations of HS cells. Changing the simulated distance of the background did not change the responses of any of the two cell types. Only if the signals of the FD1b cells of both optic lobes were subtracted the response decreased with increasing background proximity. 
FD1b-response - Changes
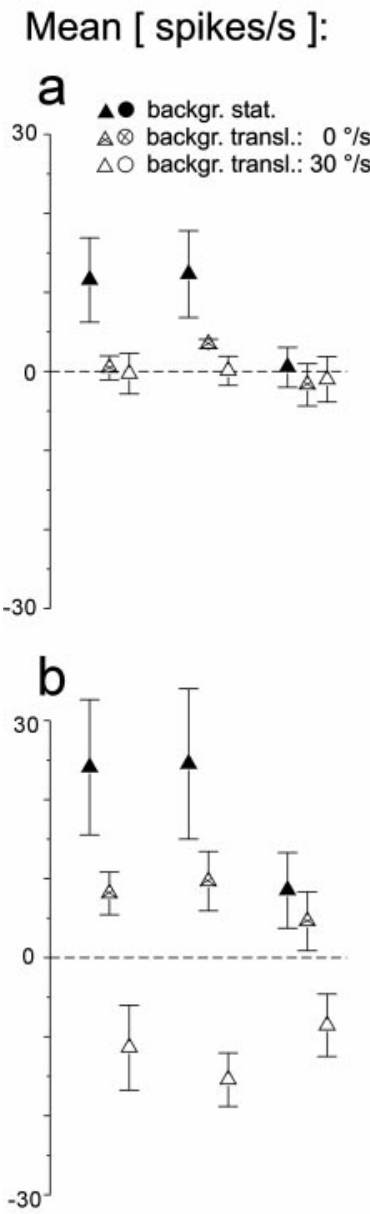

CCG (Peak):

C
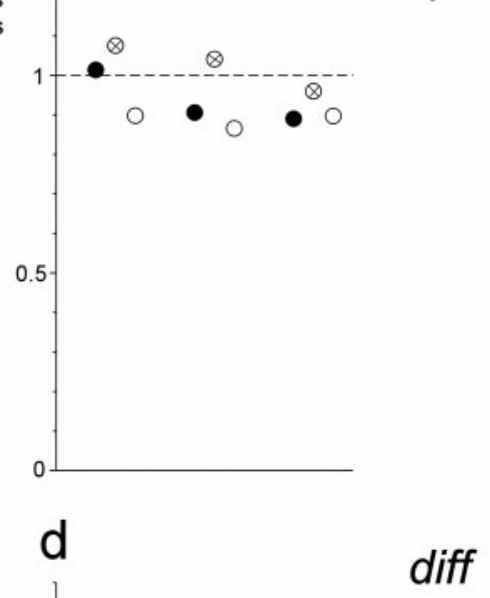

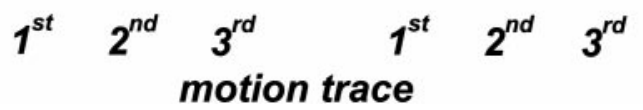

\section{HS-response - Changes}
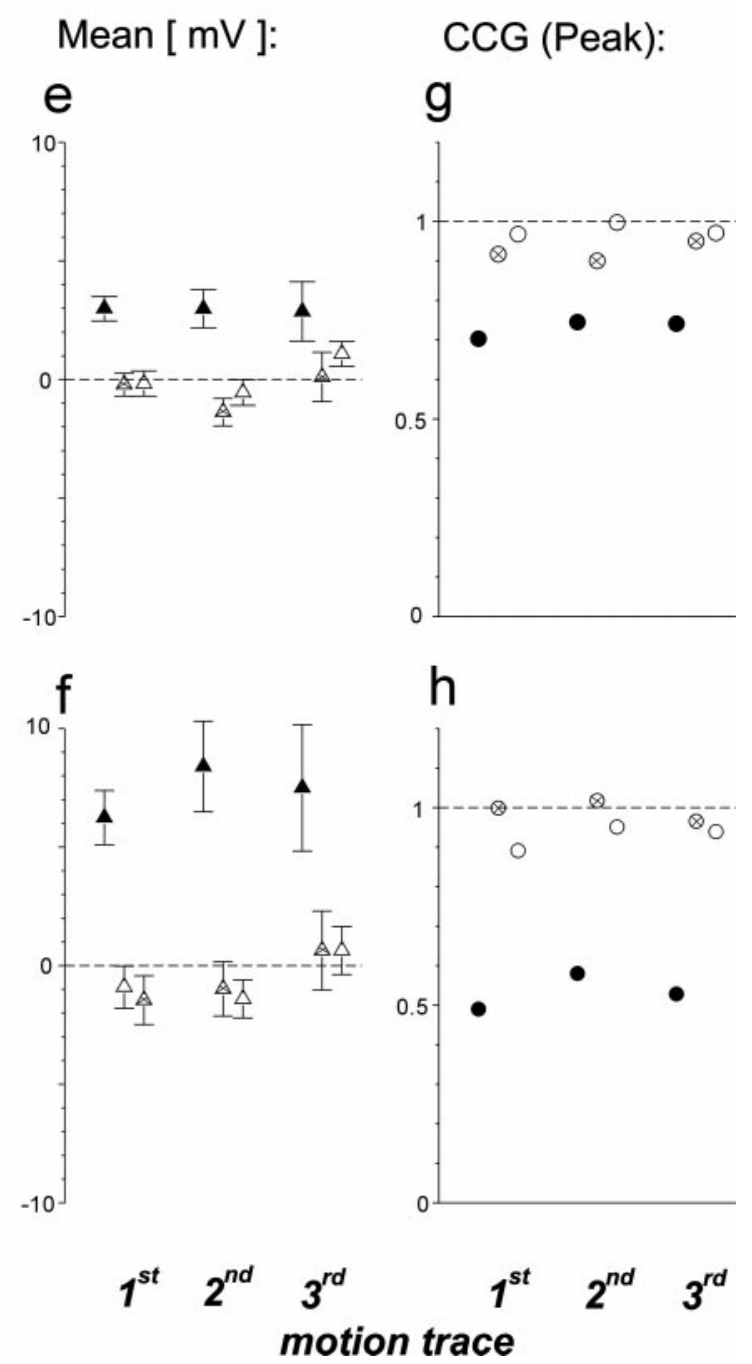

Figure 8. Quantification of the influence of background translation. Data refer to three different motion traces originating from behavioral experiments in which the same spatial situation (close object, distant background) was simulated. Filled symbols, Changes during the period of object fixation attributable to stopping background motion. Open symbols with cross, Changes caused by simulating the background at infinite distance by removing its translational velocity. Open symbols, Changes caused by simulating the background more closely by increasing its translational velocity. $a-h$, Data analysis as described for Figure 7. $n=5$ cells $(a-d) ; n=4$ cells $(e-h)$. Error bars indicate SEM.

\section{DISCUSSION}

Motion-sensitive cells presumably involved in object detection (FD1b cells) and cells of the compensatory optomotor system (HS cells) were confronted with optic flow as experienced by a behaving fly engaged in object fixation. FD1b cells responded very specifically to object motion. The responses were to a large extent independent of concurrent background motion. This finding was surprising, given the fact that FD1b cells are most likely inhibited by neurons most sensitive to large-field motion. In contrast, the responses of HS cells depended on both object and background motion. Whereas object motion had a stronger influence on the temporal modulation of the HS response than background motion, background motion had a stronger influence on the average response level than object motion. Subtraction of the signals of both optic lobes led to a decrease in the specificity of FD1b cells for object motion and to an increase of the specificity of the HS cells for background motion.

\section{Classification of FD1b cells}

FD cells were first described by Egelhaaf (1985b). Introducing a new classification system, Gauck and Borst (1999) subdivided FDlike cells according to whether and how strong they are inhibited during ipsilateral large-field motion. Ipsilateral inhibition, however, might be masked by the ipsilateral excitatory input if the horizontal extent of the motion stimulus is not sufficiently large (Kimmerle and Egelhaaf, 2000). This notion is corroborated by the finding that ipsilateral inhibition was much stronger in the present as compared to a previous study on the FD1b cell, in which we used stimuli with a smaller horizontal extent (Kimmerle and Egelhaaf, 2000, their Fig. 1, compare $b$, second box with $a$, second box; the shift of the median of the $R_{\mathrm{LF}} / R_{\mathrm{SF}}$ distributions amounts to 0.53 ). Because all FD1b cells recorded in the present study received inhibitory input from both the ipsilateral and the contralateral visual field and because the strength of binocular and of ipsilateral inhibition was unimodally distributed, we suggest that FD1b cells form a homogeneous class of cells that cannot be further subdivided on the basis of their presently known properties. FD1b cells are likely to belong to the so-called rCI-IIa cells of the Gauck and Borst (1999) classification scheme.

\section{Replay of behaviorally generated optic flow}

In the present study behavioral and cellular responses were not recorded simultaneously. Electrophysiological recordings in behaving animals are possible in some behavioral paradigms, for instance, in monkeys (Newsome et al., 1989; Gallant et al., 1998; 
Vinje and Gallant, 2000) (for review, see Newsome, 1997) but can hardly be achieved in flying flies (but see Heide, 1983). Can the cellular responses recorded in the present replay experiments be regarded as equivalent to the responses in behavioral situations? Replaying behaviorally generated optic flow in a behavioral situation in the flight simulator under open-loop conditions induces weaker responses of the flies than in the preceding closed-loop situation (Heisenberg and Wolf, 1988). However, there is evidence that the latter effect is attributable to signal processing at a stage subsequent to the motion-sensitive TCs: (1) the responses of another fly TC (the $\mathrm{H} 1$ cell) are the same in a tethered flying fly and in a fixed fly (Heide, 1983). (2) The response variability of TCs is much smaller than behavioral variability. Bimodal response distributions as found in object fixation behavior (Kimmerle et al., 2000) could not be found in any $\mathrm{TC}$ so far. In model simulations a considerable amount of noise had to be added to the output of a single pair of TCs to simulate realistic optomotor behavior in the fly (Warzecha and Egelhaaf, 1996). (3) So far, there is no evidence that the responses of TCs are influenced by other sensory pathways than the visual pathway or by nonvisual signals from the central parts of the fly brain. Therefore, the responses of the FD1b and HS cells recorded in replay experiments are considered to be indicative of the responses of these cells in a behavioral situation.

\section{Object and background specificity}

HS cells responded rather specifically to background motion, i.e., they were more strongly activated before the object appeared than during object fixation (Fig. $5 c$ ). This can be explained by the fact that, when the fly tried to turn toward the object, the background rotated in the opposite (the HS cells' anti-preferred) direction. This counter-rotation also explains why stopping background motion during the period of object fixation led to an increase in the average membrane potential of the HS cells (Fig. 7e). The HS cells were also influenced by object motion because the object was moving within their receptive field. Consequently, not displaying the object led to a stronger hyperpolarization. During the period of object fixation the average responses of the HS cells did not depend on background translation, at least for the tested translational velocities (Fig. 8e). This finding cannot be explained on the basis of the response properties of HS cells which were, so far, determined with constant velocity motion (Hausen, 1982b; Horstmann et al., 2000).

In contrast to HS cells, FD1b cells responded specifically to object motion and only weakly during background motion alone (Fig. $5 a$ ). This finding is most likely the consequence of inhibitory input FD1b cells receive from TCs sensitive to large-field motion. During object fixation the responses of FD1b cells were mainly determined by object motion, whereas background motion had little influence on the cellular response (Figs. 7a,c, 8a,c). This property is by no means trivial, because object selectivity of the FD1b cell is based on inhibition during background motion (Fig. 1). The inhibitory elements might themselves be inhibited during object fixation and concurrent background counterrotation. Moreover, the FD1b cells were not only affected by background motion via inhibitory large-field elements but also directly by their retinotopic input. The relative independence of the responses of FD1b cells from background motion during object fixation is probably a consequence of a balance between excitation and inhibition mediated by the retinotopic input as well as between inhibition and disinhibition mediated by large-field elements. Thus, although object selectivity is attributable to inhibitory input from elements tuned to large-field motion, the neuronal circuitry seems to be organized such that the influence of background motion on FD1b cell firing is reduced to a minimum in behaviorally relevant situations.

\section{Processing of the signals of both optic lobes}

Optomotor course stabilization in flies has been suggested to result from a subtraction of signals mediated by motion-sensitive cells in the right and the left optic lobe (Götz, 1975). As a working hypothesis, we initially assumed that further processing of both the HS and FD1b responses might involve subtraction (see Materials and Methods). For both cell types the difference signals were more strongly influenced by background motion than the responses of the respective ipsilateral cell alone. Course stabilization is accomplished by a reduction of global rotational movements. Assuming that HS cells play a central role in this behavioral context, their increasing specificity for background motion resulting from signal subtraction appears advantageous and supports the hypothesis of Götz (1975). Subtraction of the heterolateral neuronal signals could be realized by linear transmission and a simple symmetrical connection to the flight motor without lateral interactions. For object fixation, a stronger influence of background motion cannot be considered supportive. Therefore, subtraction does not appear a suitable way to integrate the signals of the FD1b cells of both optic lobes. We suggest that transmission of the FD1b signals to the motor system may involve lateral inhibitory interactions and/or nonlinearities such as a threshold. Such mechanisms could avoid the high selectivity for object motion of the FD1b cell being compromised by the respective contralateral cell that is not subjected to object motion.

\section{General implications}

Cells sensitive to relative motion between an object and its background have been found in different species (see introductory remarks). In primates, the responses of such cells located in cortical area MT are further integrated on a higher processing level in area MST. Cells in different regions of area MST are sensitive to optic flow as might occur during self-motion as well as to object motion (Tanaka et al., 1993; Duffy and Wurtz, 1995; Britten and Wezel, 1998). Accordingly, area MST has been suggested to play a central role in navigation and figure-ground segregation. So far MST cells have been characterized mainly with artificially designed optic flow stimuli. The present study underlines the significance of behaviorally generated stimuli when assessing the characteristics of visual interneurons. FD cells are likely to be key elements in figureground discrimination during flight and appear thus suited to guide the fly's approach toward objects of potential interest (e.g., landing sites). When confronted with stimuli as occur in a behavioral situation during object fixation, the FD1b cells of the fly show a high degree of object specificity and relative invariance to background motion. We conclude that the use of more naturalistic stimuli in electrophysiological experiments promises a deeper insight into the functional role and performance of nerve cells in real life.

\section{REFERENCES}

Allman J, Miezin F, McGuinness E (1985) Direction- and velocity-specific responses from beyond the classical receptive field in the middle temporal visual area (MT). Perception 14:105-126.

Borst A (1990) How do flies land ? From behavior to neuronal circuits Bio Science 40:292-299.

Britten KH, Wezel RJA van (1998) Electrical microstimulation of cortical area MST biases heading perception in monkeys. Nat Neurosci 1:59-63.

Collett TS (1971) Visual neurones for tracking moving targets. Nature 232:127-130.

Duffy CJ, Wurtz RH (1995) Responses of monkey MST neurons to optic flow stimuli with shifted centers of motion. J Neurosci 15:5192-5208.

Egelhaaf M (1985a) On the neuronal basis of figure-ground discrimination by relative motion in the visual system of the fly. I. Behavioural constraints imposed on the neuronal network and the role of the optomotor system. Biol Cybern 52:123-140.

Egelhaaf M (1985b) On the neuronal basis of figure-ground discrimination by relative motion in the visual system of the fly II. Figure-detection cells, a new class of visual interneurons. Biol Cybern 52:195-209.

Egelhaaf M, Borst A (1993) A look into the cockpit of the fly: visual orientation, algorithms and identified neurons. J Neurosci 13:4563-4574.

Egelhaaf M, Warzecha A-K (1999) Encoding of motion in real time by the fly visual system. Curr Opin Neurobiol 9:454-460. 
Frost BJ, Nakayama K (1983) Single visual neurons code opposing motion independent of direction. Science 220:744-745.

Gallant JL, Connor CE, Van Essen DC (1998) Neural activity in areas V1, V2 and V4 during free viewing of natural scenes compared to controlled viewing. NeuroReport 9:2153-2158.

Gauck V, Borst A (1999) Spatial response properties of contralateral inhibited lobula plate tangential cells in the fly visual system. J Comp Neurol 406:51-71.

Götz KG (1975) The optomotor equilibrium of the Drosophila navigation system. J Comp Physiol 99:187-210.

Hausen K (1982a) Motion sensitive interneurons in the optomotor system of the fly. I. The horizontal cells: Structure and signals. Biol Cybern 45:143-156.

Hausen K (1982b) Motion sensitive interneurons in the optomotor system of the fly. II. The horizontal cells: receptive field organization and response characteristics. Biol Cybern 46:67-79.

Hausen K, Egelhaaf M (1989) Neural mechanisms of visual course control in insects. In: Facets of vision (Stavenga D, Hardie R, eds) pp 391-424. Berlin: Springer.

Heide G (1983) Neural mechanisms of flight control in Diptera. In: Bionareport 2 (Nachtigall W, ed), pp 35-52. New York: Stuttgart.

Heisenberg M, Wolf R (1988) Reafferent control of optomotor yaw torque in Drosophila melanogaster. J Comp Physiol [A] 163:373-388.

Horstmann W, Egelhaaf M, Warzecha A-K (2000) Synaptic interactions increase optic flow specificity. Eur J Neurosci 12:2157-2165.

Kimmerle B, Egelhaaf M (2000) Detection of object motion by a fly neuron during simulated flight. J Comp Physiol [A] 186:21-31.

Kimmerle B, Egelhaaf M, Srinivasan MV (1996) Object detection by relative motion in freely flying flies. Naturwissenschaften 83:380-381.

Kimmerle B, Warzecha A-K, Egelhaaf M (1997) Object detection in the fly simulated translatory flight. J Comp Physiol [A] 181:247-255.

Kimmerle B, Eikermann J, Egelhaaf M (2000) Object fixation by the fly during tethered flight in a simulated 3D-environment. J Exp Biol 203:1723-1732.

Land MF, Collett TS (1974) Chasing behaviour of houseflies (Fannia canicularis). A description and analysis. J Comp Physiol [A] 89:331-357.

Newsome WT (1997) Deciding about motion: linking perception to action. J Comp Physiol [A] 181:5-12.

Newsome WT, Britten KH, Movshon JA (1989) Neuronal correlates of a perceptual decision. Nature 341:52-54.

Reichardt W, Poggio T (1979) Figure-ground discrimination by relative movement in the visual system of the fly. Part I: Experimental results. Biol Cybern 35:81-100.

Sterling P, Wickelgren BG (1969) Visual receptive fields in the superior colliculus of the cat. J Neurophysiol 32:1-15.

Tanaka K, Hikosaka K, Saito H, Yukie M, Fukada Y, Iwai E (1986) Analysis of local and wide-field movements in the superior temporal visual areas of the macaque monkey. J Neurosci 6:134-144.

Tanaka K, Sugita Y, Moriya M, Saito H-A (1993) Analysis of object motion in the parietal part of the medial superior temporal area of the macaque visual cortex. J Neurophysiol 69:128-142.

Vinje WE, Gallant JL (2000) Sparse coding and decorrelation in primary visual cortex during natural vision. Science 287:1273-1276.

Virsik RP, Reichardt W (1976) Detection and tracking of moving objects by the fly Musca domestica. Biol Cybern 23:83-98.

Wagner H (1982) Flow-field variables trigger landing in flies. Nature 297:147-148.

Wagner H (1986) Flight performance and visual control of flight of the free-flying housefly (Musca domestica L.). II. Pursuit of targets. Philos Trans R Soc Lond B Biol Sci 312:553-579.

Warzecha A-K, Egelhaaf M (1996) Intrinsic properties of biological motion detectors prevent the optomotor control system from getting unstable. Philos Trans R Soc Lond B Biol Sci 351:1579-1591. 